\title{
Quasi-One-Dimensional Quantum Ferrimagnets
}

\author{
R. R. Montenegro-Filho and M. D. Coutinho-Filho \\ Laboratório de Física Teórica e Computacional, Departamento de Física, \\ Universidade Federal de Pernambuco, 50670-901, Recife, PE, Brasil
}

\begin{abstract}
We present an exact diagonalization study of the half-filled Hubbard model on bipartite quasione-dimensional lattices. In particular, we emphasize the dependence of the ferrimagnetic ground state properties, and its associated magnetic excitations, on the Coulomb repulsion $U$.
\end{abstract}

PACS numbers: 71.10.Fd, 75.10.Lp, 75.10.Jm, 75.50.Gg

Keywords: Hubbard Model, Heisenberg Model, Ferrimagnetism, Magnetic Polymers

\section{INTRODUCTION}

In the last years the physics of quasi-one-dimensional compounds has been the object of intense theoretical and experimental investigations. In this work we study the ground state (GS) and magnetic excitations of two bipartite chains motivated by low-dimensional inorganic [1] and organic [2] ferrimagnets: the $A B_{2}$ chain in Fig. [1(a) and the $A B C$ chain in Fig. 廿(b), respectively. We shall use the simplest approach for interacting electron systems on a lattice with $N$ sites, namely the one-band Hubbard Model (HM):

$$
\mathcal{H}=-t \sum_{\langle i, j\rangle, \sigma} c_{i \sigma}^{\dagger} c_{j \sigma}+U \sum_{i} n_{i \uparrow} n_{i \downarrow}
$$

where $c_{i \sigma}^{\dagger}\left(c_{i \sigma}\right)$ is the creation (annihilation) operator for electrons with spin $\sigma$ at site $i ; t$ is the hopping parameter and $U$ is the on site Coulomb repulsion. For the extremely localized regime $(U \gg t)$ and $n \equiv N_{e} / N=1$, where $N_{e}$ is the number of particles, the HM can be mapped onto the Antiferromagnetic Heisenberg Model (AHM) [3]:

$$
\mathcal{H}=\frac{J}{2} \sum_{\langle i, j\rangle} \vec{S}_{i} \cdot \vec{S}_{j}
$$

where $J=4 t^{2} / U$. We should mention that Macêdo et al. [4], using a variety of numerical methods, have derived the ferrimagnetic nature of $A B_{2}$ chains modeled by the HM in the strong and weak coupling regimes. Here we focus on the half-filled band case $(n=1)$ and extend these previous studies to much larger systems using Lanczos exact diagonalization technique.

It is also worth mentioning that the two lattices present three electronic bands in the tight-binding limit $(U=0)$ : one flat band at $\epsilon=0$ for the two chains; and two dispersive ones, $\epsilon(q)= \pm 2 t \sqrt{2} \cos (q / 2)$ and $\epsilon(q)= \pm t \sqrt{1+[2 \cos (q / 2)]^{2}}$, with $q=2 \pi l /(N / 3)$ and $l=0 \ldots(N / 3)-1$ for the $A B_{2}$ and $A B C$ chains, respectively. The flat band is closely associated with ferrimagnetic properties of both chains at half-filling [4, 5]. A particular feature of the $A B_{2}$ chain is a local invariance under the exchange of the $B$ sites in any cell $l[\underline{6}]$. The eigenvalues of the exchange operator being $p_{l}= \pm 1$. This symmetry leads to a conserved local spatial parity that in the Heisenberg limit asserts that the $B$ sites form either a singlet $\left(p_{l}=1\right)$ or a triplet $\left(p_{l}=-1\right)$ bond state. Therefore for the symmetry sector in which all $p_{l}=-1$ the spectrum is identical to that of the alternating spin- $\frac{1}{2} / \operatorname{spin}-1$ AHM chain $[\underline{6}]$.

\section{FERRIMAGNETIC GROUND STATE}

A theorem due to Lieb and Mattis [] asserts that the GS of the AHM on a bipartite lattice has total spin $S_{g}=\left|N_{1}-N_{2}\right| / 2$, where $N_{1}$ and $N_{2}$ are the number of lattice sites at the sub-lattices 1 and 2, respectively. So, if $\left|N_{1}-N_{2}\right| \sim N$ the system display unsaturated ferromagnetic (F) GS. The coexistence of both $\mathrm{F}$ and antiferromagnetic (AF) long-range order implies in ferrimagnetism, as rigorously proved by Tian [8]. Another crucial step was provided by Lieb [9], who proved that the GS of the HM for $n=1$ has $S_{g}=\left|N_{1}-N_{2}\right| / 2$ for any $U>0$. In this case, Tian and collaborators [10] further established the ferrimagnetic (FERRI) long-range order of the GS if $\left|N_{1}-N_{2}\right| \sim N$. The unit cell of the two chains of interest has 2 sites in one sublattice and 1 in the other (see Fig. (1), so that $S_{g}=N_{c} / 2$, where $N_{c}=N / 3$ is the number of unit cells. However, although ferrimagnetism is expected, the specific magnetic structure of a unit cell may strongly depend on the Coulomb coupling, as well as on quantum fluctuations, and is not known a priori from the more general results of the theorems stated above. 
We can probe the magnetic order through the Magnetic Structure Factor (MSF):

$$
S(q)=\frac{1}{N} \sum_{i, j} e^{i q\left(x_{i}-x_{j}\right)}\left\langle\vec{S}_{i} \cdot \vec{S}_{j}\right\rangle
$$

which is related to the zero-field static magnetic susceptibility by $\chi(q=0)=S(0) /\left(k_{B} T\right)$, where $k_{B}$ is the Boltzmann constant and $T$ is the temperature. The condition for a $\mathrm{F}$ (AF) ordered state is that $S(0)[S(\pi)] \sim N$, so that in a long-range FERRI GS the two conditions must be fulfilled. This is indeed the case for the two chains, as shown in Figs. 1] and 2] both in the strong and weak coupling limits.
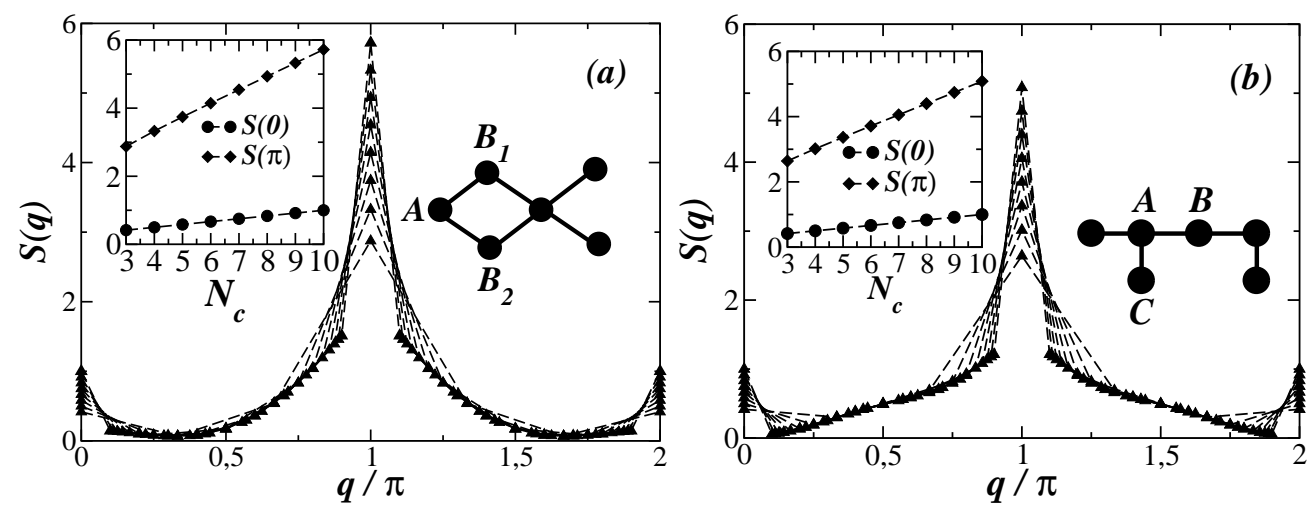

FIG. 1: Magnetic Structure Factor $S(q)$ for the $A B_{2}$ (a) and $A B C$ (b) chains in the Heisenberg limit $(U \gg t)$. The size ranges from $N_{c}=3$ to $N_{c}=10$. The insets display the size dependence of the Ferromagnetic $[S(0)]$ and Antiferromagnetic $[S(\pi)]$ peaks. Dashed lines are guides for the eye.
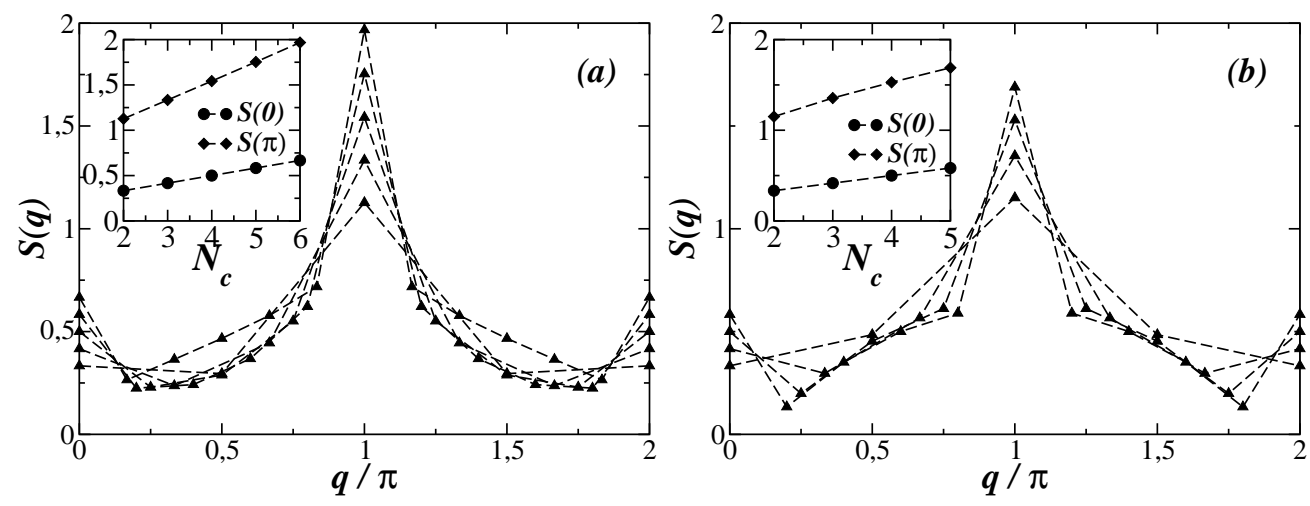

FIG. 2: Magnetic Structure Factor $S(q)$ for the $A B_{2}$ (a) and $A B C$ (b) chains using the HM for $U=2 t$. The size ranges from $N_{c}=2$ to $N_{c}=6$ for the $A B_{2}$ chain and to $N_{c}=5$ for the $A B C$. The inset presents the size dependence of the Ferromagnetic $[S(0)]$ and Antiferromagnetic $[S(\pi)]$ peaks. Dashed lines are guides for the eye.

Due to the critical nature of both chains at low temperatures, the correlation length $\xi$ and $\chi(q=0)$ satisfy power law behaviour: $\xi \sim T^{-\nu}$ and $\chi \sim T^{-\gamma}$ as $T \rightarrow 0$. Since $\xi \sim N$ at $T=0$, using scaling arguments and the results of Fig. 11 we have $T^{-\gamma} \sim T^{-\nu} / T$, i. e., $\gamma-\nu=1$, in agreement with the values $\gamma=2$ and $\nu=1$ derived using renormalization group techniques [1]].

In Fig. 3] we present the local magnetization $\left(S_{i}^{z}\right)$ and the average local value of double occupancy $\left(\left\langle n_{i \uparrow} n_{i \downarrow}\right\rangle\right)$ as function of $U$. Since the system has a spontaneously broken $S U(2)$ symmetry, we can choose the spin sector $S^{z}=S_{g}$ to study these quantities, where $S^{z}$ is the total spin $z$ component. In the Heisenberg limit $\left(U \gg t,\left\langle n_{i \uparrow} n_{i \downarrow}\right\rangle=0\right)$, the bulk magnetization estimated from chains up to $N_{c}=10$ are: $\left\langle S_{A}^{z}\right\rangle=-0.2925$ and $\left\langle S_{B_{1,2}}^{z}\right\rangle=0.3962$, for the $A B_{2}$ chain, in agreement with density matrix renormalization group (DMRG) calculations [12] and with values for $\left\langle S_{A}^{z}\right\rangle$ and $2\left\langle S_{B_{1,2}}^{z}\right\rangle$ in the spin- $\frac{1}{2} /$ spin-1 chain [13]. For the $A B C$ chain, $\left\langle S_{A}^{z}\right\rangle=-0.2567,\left\langle S_{B}^{z}\right\rangle=0.3741$ and $\left\langle S_{C}^{z}\right\rangle=0.3826$. 
Notice that a FERRI structure sustain quantum fluctuations, which strongly reduce the local magnetization from the classical value, although in any case the unit cell magnetization remains $1 / 2$. In the non-interacting limit $(U=0)$ we see in Figs. 3(a) and 3 (b) that $\left\langle S_{A}^{z}\right\rangle=0$ and $\left\langle S_{B_{1}(B)}^{z}+S_{B_{2}(C)}^{z}\right\rangle=0.5$ for the $A B_{2}(A B C)$ chain. These results being related to the band structure: filling the bands up to $n=1$ and choosing the spin sector $S^{z}=S_{g}$, there exist $N_{c}$ unpaired electrons in the flat band which occupy $B_{1}(B)$ and $B_{2}(C)$ sites only. It is important to note that in this limit the global GS does not have an unique value of $S$, but rather $S \leq N_{c} / 2$. As the Coulomb coupling is turned on, the GS, with a unique $S_{g}=N_{c} / 2$, evolves adiabatically from the weak to the strong coupling regime (Fig. 3).

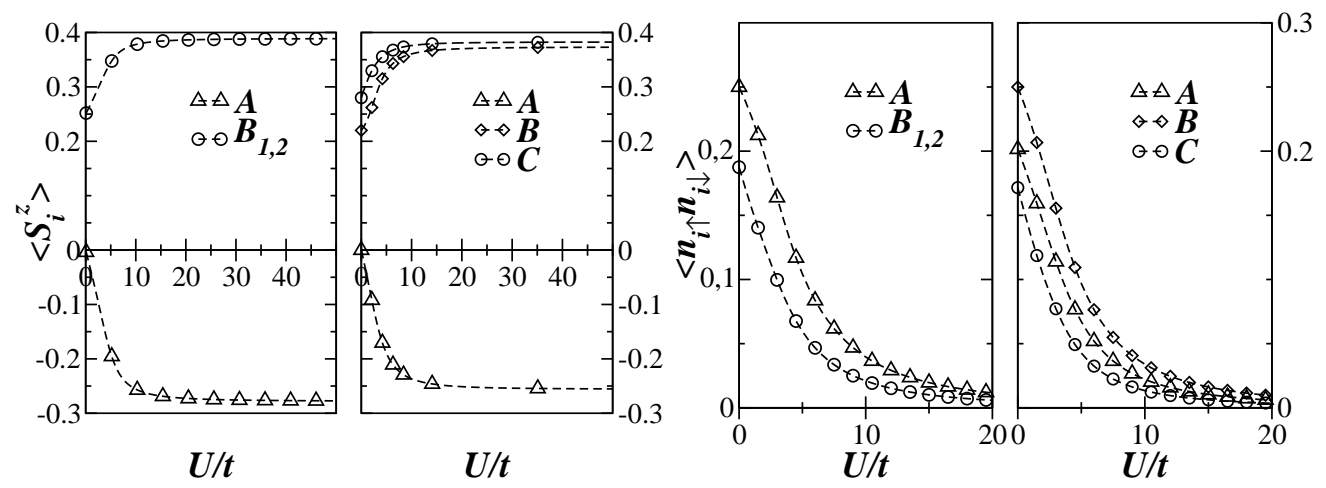

FIG. 3: Site magnetization $\left\langle S_{i}^{z}\right\rangle$ and average site double occupancy $\left\langle n_{i \uparrow} n_{i \downarrow}\right\rangle$ as function of $U$ for $N_{c}=5$ in the $A B_{2}$ and $A B C$ chains in the spin sector $S^{z}=S_{g}$. Dashed lines are guides for the eye.

\section{MAGNETIC EXCITATIONS}

Systems with a FERRI GS naturally have F (lowering the GS spin) and AF (rising the GS spin) magnons as their elementary magnetic excitations. The two chains have three spin wave branches: an AF mode, defined as $\Delta E_{S+}(q)=E\left(S^{z}=S_{g}+1, q\right)-E_{0}$; and two F ones, derived from $\Delta E_{S-}(q)=E\left(S^{z}=S_{g}-1, q\right)-E_{0}$, where $E_{0}$ is the GS energy and $E\left(S^{z}, q\right)$ are lowest excitation energies in the sector $\left\{S^{z}, q\right\}$, with $q=2 \pi l / N_{c}$ and $l=0,1, \ldots, N_{c} / 2$. The acoustical and optical $\mathrm{F}$ modes are identified from symmetry considerations. These modes are depicted in Fig. III for the two chains using the AHM: the AF mode has a gap, denoted by $\Delta_{S+}$; the F1 mode is gapless, i. e., the Goldstone mode, consistent with the symmetry broken phase of the chains; the gap of the F2 mode is denoted by $\Delta_{S-\text {. }}$

For the $A B_{2}$ chain the gapped F2 branch is flat, Fig. III(a), and the other modes are dispersive. Since these dispersive modes preserve the local triplet bond, they are identical to those found in the spin- $\frac{1}{2} / \operatorname{spin}-1$ [13] chain. Surprisingly, Linear Spin Wave Theory (LSWT) [14] predicts that $\Delta_{S-}=1$, very close to our estimated value: $\Delta_{S-}=1.0004 \mathrm{~J}$. Moreover, a good agreement is found for the gapless F1 branch in the long wave-length limit. However, both LSWT and mean field (MF) theory [15] predicts $\Delta_{S+}=1$, deviating from our estimated exact diagonalization value: $\Delta_{S+}=1.7591 \mathrm{~J}$, which is in excelent agreement with numerical and analytical calculations for the spin- $\frac{1}{2} /$ spin-1 chain [13]. On the other hand, the Interacting Spin Wave Theory [16] derives a better result for $\Delta_{S+}$, but it implies in a higher shift for $\Delta_{S-}$ (flat mode) not observed in our data of Fig. III(a). This flat mode indicates the localized nature of the excitation, which is associated with the formation of a singlet state (even parity symmetry) between the $B$ sites in one cell, while the other cells have odd parity symmetry ( $B$ sites in a triplet state). Thus, since the symmetries of the F modes (dispersive and flat) are distinct, a possible level crossing is not avoided, as in fact observed in the data of Fig. III)(a): for $q \gtrsim 0.75 \pi$ the localized excitation is below the dispersive one.

For the $A B C$ chain we can see in Fig. III(b) that the three branches are dispersive. Like in the $A B_{2}$ chain, the AF mode is gapped with $\Delta_{S+}=1.4189$. The LSWT [17] conducts to a qualitative but not quantitative correct AF branch. However, a good agreement is found for the gapless F1 mode in the long-wavelength limit. Moreover, the gap for the F2 mode is estimated to be $\Delta_{S-}=0.6778 \mathrm{~J}$. We can clearly observe in Fig. III (b) the level repulsion between the $\mathrm{F}$ branches, which in this case have the same symmetry. 

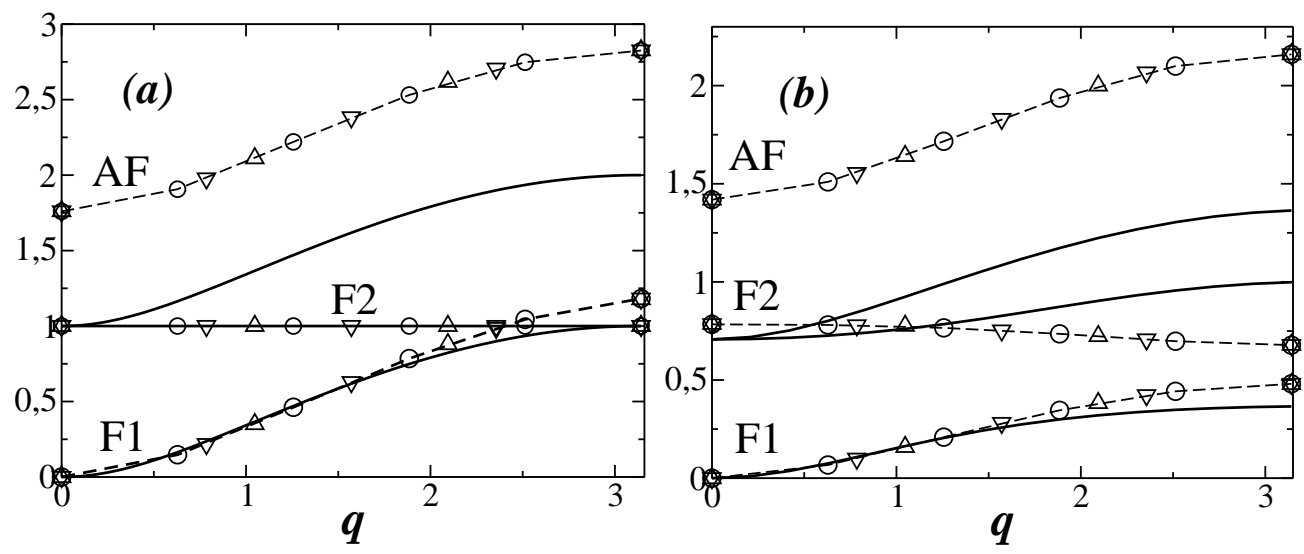

FIG. 4: $\mathrm{F}$ and $\mathrm{AF}$ spin wave modes of the $A B_{2}$ (a) and $A B C$ (b) chains for $N_{c}=10$ (circles), 8 (triangles down), 6 (triangles up). Solid lines are the LSWT results from Ref. [14] $\left(A B_{2}\right)$ and Ref. [17] $(A B C)$; dashed lines are guides for the eye.

\section{MAGNETIZATION PLATEAUS AND SPIN WAVE GAPS}

The AF gaps found above are responsible for plateaus in the magnetization per spin $\left[m(H)=\left\langle S^{z}\right\rangle / N\right]$ as function of the applied magnetic field $H$. In fact, it has been shown [18] that if $f(s-m)=$ integer, a plateau may appear in the magnetization curve of the AHM. In the last equation, $s$ is the site total spin, $m$ is the magnetization and $f$ is the number of sites in one unit period of the GS for a given value of $H$. The Hamiltonians that we are considering here have $s=1 / 2$ and three sites per unit cell, so, unless the system spontaneously breaks the translation invariance (e.g., by growing the unit cell), we expect a plateau at $m=1 / 6$. This is indeed observed in Fig. 5 and its width matches exactly $\Delta_{S+}$, which measures the region of stability of the FERRI phase. For higher fields the magnetization increases in the expected way [18], as shown in the inset (2) of Figs. [5 (a) and [5 (b), before the magnetization saturates at $m=1 / 2$ for $H=3 J$ and $H=2.3660 J$, respectively. This field dependent behaviour contrasts with the linear one predicted by MF theory [15].
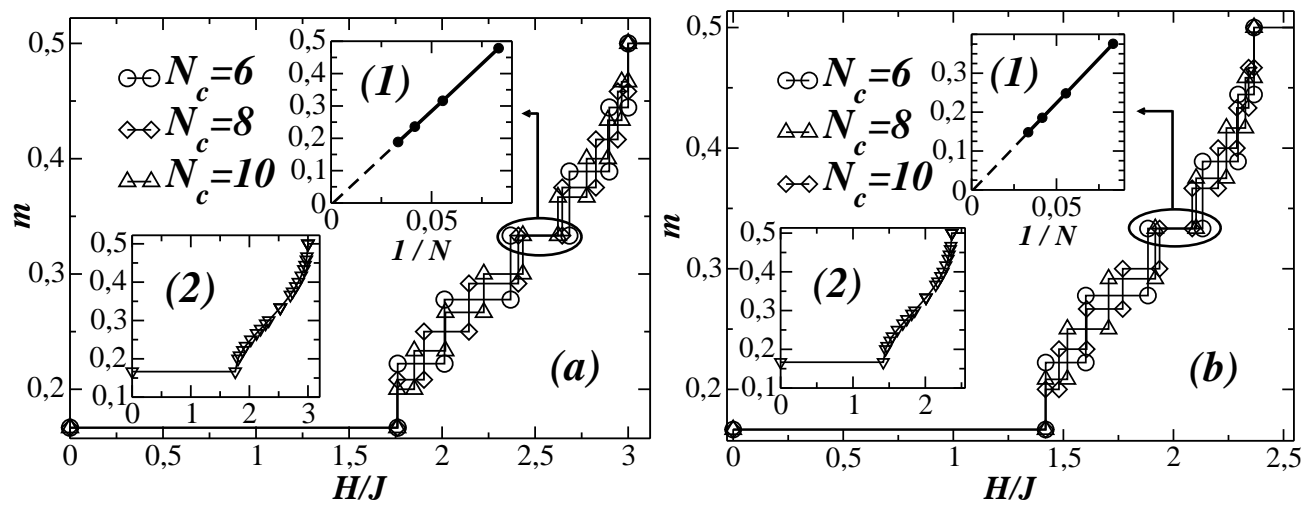

FIG. 5: Magnetization as function of the applied magnetic field $H$ for the $A B_{2}$ (a) and $A B C$ (b) chains using the AHM. The inset (1) presents a finite size scaling study for $m=1 / 3$; the inset (2) is a curve traced from the midpoints of the steps using the finite size results, except at plateau regions.

Now we turn to study the evolution of the AF and the F2 spin wave gaps as function of $U$ using the HM: $\Delta_{S+}(U)$ (plateau width) and $\Delta_{S-}(U)$, respectively. In order to reduce finite size effects in calculating $\Delta_{S \pm}(U)$ we have used the boundary condition that minimizes the gap between the GS and excited levels. Our estimated curves are depicted in Fig. [6] using the scaling ansatz [19] for extrapolation: $\Delta=\Delta_{\infty}+c_{1} N^{-2}+c_{2} N^{-4}$, with $N=9,12,15$ and 18 for the $A B_{2}$ chain; $N=6,9,12$ and 15 for the $A B C$ chain. Note that $\Delta_{S+}(U=0)=0$ and $2 t$ for the $A B_{2}$ and $A B C$ chains, respectively. This can be understood by noticing that $\Delta_{S+}(U=0)$ is given by the gap between the two dispersive 
tight-binding electronic bands. Moreover, $\Delta_{S+}(U \rightarrow 0) \sim U^{x}, x \simeq 2$, for the $A B_{2}$ chain, whereas for the $A B C$ chain $\Delta_{S+}(U \rightarrow 0)$ increases linearly before reaching the tight-binding limit $2 t$. For both chains, we observe a crossover to the Heisenberg limit: $\Delta_{S+} \sim J$, in agreement with the results of Fig. III Further, we notice that $\Delta_{S-}(U \rightarrow 0)$ nullifies linearly with $U$, for both chains, following the behaviour of the gap between the flat electronic bands 4$]$.

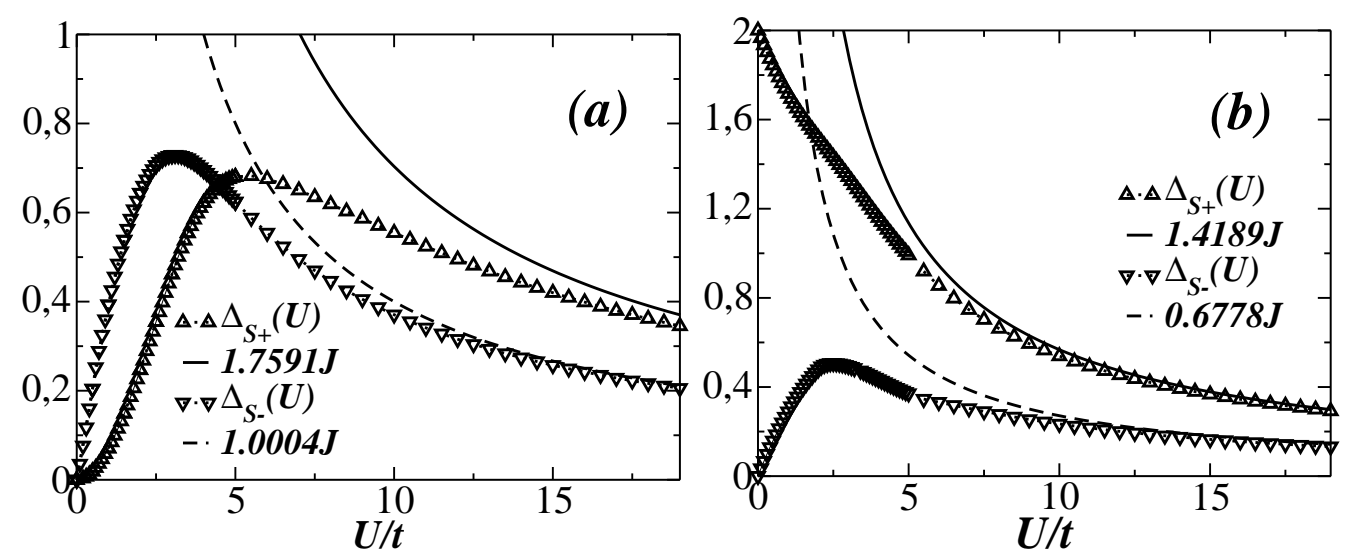

FIG. 6: Extrapolated AF $\left(\Delta_{S+}\right)$ and F2 $\left(\Delta_{S_{-}}\right)$spin wave gaps in units of $t$ for the $A B_{2}$ (a) and $A B C$ (b) chains as function of $U$ using the HM. Solid (dashed) line is the AF (F2) gap for the AHM with $J=4 t^{2} / U$; dotted lines are guides for the eye.

\section{CONCLUSIONS}

In this work we reported a detailed exact diagonalization study of the half-filled Hubbard model on bipartite quasi-one-dimensional lattices. These chains have experimental motivation in inorganic and organic ferrimagnetic polymers. We have focused on the description of their ferrimagnetic ground state properties and magnetic excitations. In particular, we have revealed the non-trivial physical behaviour exhibited by these chains as function of the Coulomb coupling strength.

\section{ACKNOWLEDGEMENTS}

We acknowledge several useful discussion with A. L. Malvezzi. This work was supported by CNPq, Finep, FACEPE and CAPES (Brazilian agencies).

[1] J. Silvestre and R. Hoffman, Inorg. Chem. 24 (1985) 4108; M. Drillon et al, J. Magn. Magn. Mater. 128 (1993) 83.

[2] Y. V. Korshak, T. V. Medvedera, A. A. Ovchinnikov and V. N. Spector, Nature 326 (1987) 370; H. Nishide, Adv. Mater. 7 (1995) 937.

[3] E. P. Raposo and M. D. Coutinho-Filho, Mod. Phys. Lett. B 9 (1995) 817.

[4] A. M. S. Macêdo, M. C. dos Santos, M. D. Coutinho-Filho, and C. A. Macêdo, Phys. Rev. Lett. 74 (1995) 1851.

[5] H. Tasaki, Prog. of Theor. Phys. 99 (1998) 489, and references therein.

[6] F. C. Alcaraz and A. L. Malvezzi, J. Phys. A: Math. Gen. 30 (1997) 767.

[7] E. H. Lieb and D. C. Mattis, J. Math. Phys. 3 (1962) 749.

[8] G.-S. Tian, J. Phys. A: Math. Gen. 27 (1994) 2305.

[9] E. H. Lieb, Phys. Rev. Lett. 62 (1989) 1201.

[10] S.-Q. Shen, Z.-M. Qiu and G.-S. Tian, Phys. Rev. Lett. 72 (1994) 1280; G.-S. Tian and T.-H. Lin, Phys. Rev. B 53 (1996) 8196.

[11] E. P. Raposo and M. D. Coutinho-Filho, Phys. Rev. B 59 (1999) 14384; Phys. Rev. Lett. 78 (1997) 4853.

[12] G. Sierra, M. A. Martín-Delgado, S. R. White, D. J. Scalapino and J. Dukelsky, Phys. Rev. B 59 (1999) 7973.

[13] S. Yamamoto, S. Brehmer and H.-J. Mikeska, Phys. Rev. B 57 (1998) 13610; N. B. Ivanov, Phys. Rev. B 62 (2000) 3271 ; S. Yamamoto, Phys. Rev. B 69 (2004) 064426.

[14] C. Vitoriano, F. B. de Brito, E. P. Raposo and M. D. Coutinho-Filho, Mol. Cryst. Liq. Cryst. 374 (2002) 185. 
[15] C. Vitoriano, M. D. Coutinho-Filho and E. P. Raposo, J. Phys. A: Math. Gen. 35 (2002) 9049.

[16] T. Nakanishi and S. Yamamoto, Phys. Rev. B 65 (2002) 214418.

[17] F. B. de Brito, M. Sc. Dissertation, Universidade Federal de Pernambuco, 2001. For the $A B C$ an analytical solution is found only for $J_{A B}=J_{A C} / 2$, in which case the gapped $\mathrm{F}$ mode is flat.

[18] M. Oshikawa, M. Yamanaka, and I. Affleck, Phys. Rev. Lett. 78 (1997) 1984.

[19] L. Capriotti, F. Becca, S. Sorella and A. Parola, Phys. Rev B 67 (2003) 172404. 